\title{
La rappresentazione della differenza. Il ritorno di Meg March nella letteratura scritta dalle donne
}

\author{
Barbara Meazzi
}

\section{OpenEdition}

\section{Journals}

Edizione digitale

URL: http://journals.openedition.org/cei/927

DOI: $10.4000 /$ cei.927

ISSN: 2260-779X

\section{Editore}

UGA Éditions/Université Grenoble Alpes

\section{Edizione cartacea}

Data di pubblicazione: 15 maggio 2008

Paginazione: 219-230

ISBN: 978-2-84310-121-2

ISSN: 1770-9571

Notizia bibliografica digitale

Barbara Meazzi, «La rappresentazione della differenza. II ritorno di Meg March nella letteratura scritta dalle donne », Cahiers d'études italiennes [Online], 7| 2008, Messo online il 15 novembre 2009, consultato il 21 avril 2019. URL : http://journals.openedition.org/cei/927 ; DOI : 10.4000/cei.927 


\title{
LA RAPPRESENTAZIONE DELLA DIFFERENZA
}

\author{
IL RITORNO DI MEG MARCH NELLA \\ LETTERATURA SCRITTA DALLE DONNE ${ }^{*}$
}

\author{
Barbara Meazzi \\ Université de Savoie
}

Tieni sempre presente che è una donna.

Sai, no, le donne? Quelle tanto diverse da noi.

Stefania Bertola, Biscotti e sospetti ${ }^{2}$

Il sottotitolo potrebbe essere: «Ovvero le piccole donne crescono come possono ma sono sempre in circolazione, altro che Hasta siempre mujercitas $^{3}$ ", in riferimento al titolo di uno degli ultimi libri della cilena Marcela Serrano, che si rifa alla celeberrima saga delle sorelle March creata da Louise May Alcott nel $1868^{4}$, per certi versi a sua volta molto simile ai romanzi della Austen. Si pensi anche solo ad Orgoglio e pregiudizio 5 del 1813, in cui Elisabeth Bennet fatica a trovare una sua dimensione in mezzo alle sciocche sorelle, la cui unica ragione di vita è il matrimonio a tutti $i$ costi.

Nel romanzo della Alcott, come è ben noto, viene narrata la storia di quattro sorelle: Meg, la quale non concepisce la propria esistenza al di fuori dell'essere sposa e madre; Jo, la preferita da tutte le giovani lettrici, anticonformista ed indipendente; Beth, timida e virtuosa pianista, morta prematuramente di scarlattina e responsabile delle lacrime versate non solo da parenti e amici raccolti intorno al suo capezzale, ma anche da generazioni di bambine di tutto il mondo occidentale; Amy, pittrice in nuce, frivola ed arrivista, che si sposa con l'innamorato di Jo, nipote del ricchissimo vicino di casa. Il carattere delle quattro ragazze March viene delineato fin dall'indimenticabile incipit:

"Christmas won't be Christmas without any presents," grumbled Jo, lying on the rug.

"It's so dreadful to be poor!" sighed Meg, looking down at her old dress.

"I don't think it's fair for some girls to have plenty of pretty things, and other girls

* Notes p. 229.

$\infty$

Cahiers d'études italiennes, $\mathrm{n}^{\circ}$ 7, 2008, p. 219-230. 219 
nothing at all" added little Amy, with an injured sniff.

"We've got Father and Mother, and each other," said Beth contentedly from her corner'.

Le eredi di Jo, nella letteratura italiana contemporanea al femminile, sono numerose: si pensi a Rossana Campo, Nicoletta Vallorani, Anna Berra, piuttosto lontane dalle tormentate storie messe in scena dalla Fusini o dalla Sanvitale, da Clara Sereni o da Elisabetta Rasy.

Le figlie di Jo, all'insegna dell' iscrizione di un anonimo cubano che nel lontano 1762 annotava "Las muchachas de La Habana no tienen temor de Dios ${ }^{7}$ ", non solo non temono più Dio, ma nemmeno gli uomini. Si ribellano, conquistano la loro indipendenza, pagandola certo a caro prezzo, e indugiano nel sottolineare l'uguaglianza e la parità - quella "smania di far da sola ${ }^{8}$ " che animava già la Clelia di Pavese in Tra donne sole -, nei confronti degli uomini, ma anche la differenza rispetto a questi ultimi, differenza oltremodo accentuata in taluni romanzi della Santacroce, della Teodorani o sottolineata in alcuni saggi da Cinzia Tani. Che sono sempre discendenti della Alcott, però piuttosto del genere Piccole donne uccidono 9 .

C'è da chiedersi tuttavia che fine abbiano fatto i personaggi invece più simili a Meg. Già, perché la Campo, la Santacroce, la Teodorani, o ancora Margherita Oggero, Laura Grimaldi, Nicoletta Vallorani insistono sulla differenza tra uomo e donna e sulla donna diversa. Il topos dell'affermazione della differenza uomo-donna è diventato pressoché una norma, per le discendenti di Jo, per le nipotine di Simone De Beauvoir - che scandivano nei cortei «Figlia moglie e madre ne ho le ovaie quadre».

"Le brave bambine vanno in paradiso, quelle cattive vanno ovunque ", diceva un altro slogan femminista: sulle cattive bambine si è scritto molto, appunto, ma dove sono finite le brave? Quali sono le caratteristiche delle eredi di Meg, brave ragazze da marito? Come viene rappresentata la differenza tra le brave e le cattive bambine? E come vivono questa differenza le brave bambine?

Dopo un sintetico e parziale bilancio della situazione editoriale italiana, è nei romanzi di Barbara Garlaschelli che si cercherà la risposta, in particolare in Alice nell'ombra ${ }^{10}$ del 2002.

Noi che non siamo come le altre ${ }^{11}$

$\mathrm{Ci}$ sono donne normali e donne che non sono come le altre. Le donne normali, nei romanzi di Stefania Bertola, sono quelle che fanno una vita 
normale: «Si alzano presto, trangugiano un caffe di corsa e corrono al mercato per fare la spesa prima di andare al lavoro ${ }^{12}$. Alzarsi presto significa alzarsi verso le sette e mezzo, è il caso di Ginevra in Aspirapolvere di stelle:

La donna che lavora si alza alle sette e mezzo, e si fa il caffe. E poi si prende cura dell'epidermide, perché tra i venticinque e i trentacinque stanno lì in agguato le famose 'rughe di espressione', e bisogna sventarle ai primi sintomi ${ }^{13}$.

La donna che lavora non ha tempo «ma il poco che ha adora perderlo ${ }^{14} »$; sa tenere tutto sotto controllo, ha una famiglia perfetta, dei figli perfetti, una casa perfetta. Non come Carolina:

Carolina non è mai stata sposata, e come me non ha figli, ma qui finiscono i punti di contatto. Io non sono sposata perché sto aspettando di sposare Alex. Appena lui sarà disponibile, filo ad acquistare il più bianco degli abiti di crinolina. Carolina, invece, non è sposata perché sposarsi non le piace, vivere con gli uomini non le piace, allevare bambini non le piace. Le piace fidanzarsi ardentemente per brevi periodi, guardare $\mathrm{i}$ suoi gatti, [...] e cedere a violente passioni per attività manuali e pratiche. Ultimamente decora mobiletti ${ }^{15}$.

Nel momento in cui Carolina - che non è precisamente una cattiva bambina - desidera un figlio, significa che si è innamorata sul serio e che quindi è diventata una donna normale: l'happy end è allora assicurato e Carolina, come tutte le protagoniste del romanzo, finirà per essere una donna sentimentalmente appagata. Non c'erano né dubbi né suspence, a tale proposito, giacché il finale «fiori d'arancio » si intuiva fin dalla prima pagina del romanzetto.

Questa è letteratura di intrattenimento e la Bertola, spiritosa e talentuosa artigiana-scrittrice, non riesce tuttavia ad uscire dai solchi profondamente tracciati dalla maestra Carolina Invernizio. Nondimeno, grazie alla Bertola o attraverso i suoi libri, abbiamo la possibilità di renderci conto che le figlie di Meg, brave ragazze e brave spose, imperversano, leggono i libri di Lina Sotis sul bon ton e non si ribellano quasi mai, se non contro la sfortuna che impedisce loro di incontrare il principe azzurro bello e ricco.

Il fatto è che Stefania Bertola non è l'unica ad essersi cimentata nel genere «rosa » : le figlie di Meg proliferano, specialmente nelle librerie italiane, in maniera sconcertante ${ }^{16}$. Vanno a fare shopping con le sorelle, spendono e spandono ma poi si redimono e non hanno nemmeno niente a che vedere con le Amy ipergamiche, a cui è dedicato per esempio l'ultimo sforzo creativo dell'ex trasgressivo Aldo Busi il quale, abbandonati Cazzi e canguri $^{17}$, ha recentemente riscritto il pamphlet di Zsa Zsa Gábor intitolato Come accalappiare un uomo, come tenerselo stretto e come scaricarlo ${ }^{18}$, definito «il libro rosa più shocking dell'anno ${ }^{19}$ ". 
Ahimè, dopo aver imparato che uomini e donne sono costituzionalmente diversi ma legalmente uguali, dopo aver inteso che tale differenza non è una tara, una malattia, uno svantaggio o un difetto ma un dato di fatto, e dopo aver preso atto delle lotte condotte con coraggio dalle figlie di Jo, le figlie di Meg, sempre più numerose in letteratura, la fanno da padrone: invece di rivendicare l'uguaglianza, rivendicano la differenza o addirittura l'inferiorità, rispetto agli uomini. Torna in auge allora la donna un po'civettuola, emotiva, certo non più completamente sottomessa, per lo meno quando vuole fare shopping, sessualmente poco attiva ma comunque sempre più ossessionata dalla ricerca di un partner grazie al quale diventare moglie ed eventualmente madre, realizzando così il suo ideale tradizionale - non tradizionalista - di donna.

Le figlie di Jo non sono come le altre: hanno imparato a stimare loro stesse o per lo meno ci provano e rivendicano, magari anche insieme agli uomini, il diritto che ha ogni persona di scegliersi la vita che vuole vivere; le figlie di Meg, invece, si guardano allo specchio per verificare se il completino nuovo nasconda sufficientemente il giro-fianchi, sono ambiziose solo nella ricerca del marito, usano armi da donna per sgominare la concorrenza, sono - come già detto - piuttosto refrattarie al sesso e confondono vieppiù femminilità con civetteria ${ }^{20}$.

\section{Sorelle e nemiche: il lato oscuro di Meg}

Barbara Garlaschelli è un'autrice ancora ed ingiustamente poco nota, in Italia, ma è ben conosciuta invece dai cultori del giallo e del noir. Non è poi solo autrice di noir: Tre amiche e una farfalla narra per esempio la storia di un'amicizia tra ragazze, mentre in Sirena, del $2001^{21}$, l'autrice racconta i dieci mesi successivi all'incidente occorsole all'età di quindici anni quando, in seguito ad un tuffo in mare, si lesionò la quinta vertebra cervicale.

$O$ ridere o morire, raccolta di racconti del 1995, è il suo primo libro, appena ripubblicato dai tipi della Todaro: la vena ironica cui rimanda il titolo è sottesa poi anche in alcuni romanzi e caratterizza molti racconti dell'autrice; molto più rilevante è però la grande capacità di costruire agghiaccianti atmosfere e di penetrare l'oscurità della psiche dei suoi simili, o piuttosto delle sue simili, giacché molti suoi testi raccontano storie di donne ${ }^{22}$. Anzi, di piccole donne, piccole Meg, mogli e madri, protagoniste di vite normali e anche un po'banali che un evento, esterno o interno, viene a sconvolgere improvvisamente. Storie incisive, come 
quelle della tarantola maschio : "L'amò, morì ${ }^{23}$ "; storie sempre tragiche di cui le figlie di Meg sono protagoniste in quanto vittime oppure in quanto carnefici:

Terza notte di nozze.

Si svegliò e restò a contemplarla nella luce tenue della luna. [...]. La guardò a lungo.

Non sarebbe riuscito ad amarla più di così.

Cercò di immaginarsi la loro vita insieme, un tranquillo scorrere di giorni e di notti. Il lavoro, la casa, la famiglia, forse dei figli. Il Natale, le vacanze al mare, la settimana bianca. E poi di nuovo lavoro, casa, figli, Natali, vacanze, settimane bianche. Le loro due esistenze, un metronomo che avrebbe scandito sempre lo stesso ritmo. In eterno. [...] Le accarezzò dolcemente una guancia e le rotolò sopra. Lei spalancò gli occhi [...], poi gli sorrise.

Natale, vacanze, settimane bianche, figli, lavoro, casa. Figli. Lavoro. Casa. Figlilavorocasa, figlilavorocasa.

Continuò a sorridergli sino a quando il suo viso scomparve sotto il cuscino.

Figli, lavoro, casa.

Non aveva mai creduto all'amore eterno. (p. 83-84)

Sono donne che lavorano, donne normali, mogli che non sopravvivono alla terza notte di nozze, appunto, che per amore o per odio si trasformano improvvisamente in vendicatrici sanguinarie, come la protagonista del racconto "Un colpo ben assestato », quando scopre che il marito la tradisce, incoraggiato dalla di lui madre. "Siamo sposati da cinque anni, ma tra noi due nemmeno una sola notte è stata un'avventura " (p. 85), commenta la donna, voce narrante, a cui il marito ha giurato che non si trattava di un tradimento, ma solo dell'avventura "di una notte». Lei gli spara due colpi di pistola, che lui riesce ad evitare, fuggendo, malgrado la mancanza di agilità e la pinguedine. Il finale è sconcertante, come sempre:

Mi sento comunque appagata.

Due pallottole non sempre uccidono.

L'infarto sì. (p. 86)

Non sempre il marito è la causa da eliminare; ci sono talvolta mogli ingombranti e anche alcune madri invadenti, oppure affettuosamente asettiche, ossessivamente perfette:

Tutti gli aggettivi utilizzati dalla gente per descrivere Adelaide Breme erano accompagnati da un avverbio. Tremendamente affascinante. Sorprendentemente arguta. Prodigiosamente abile. Meravigliosamente dolce. Amorevolmente vezzosa. » (p. 53)

Le figlie di questi esseri perfetti sono invece naturalmente perfettibili :

Tutti gli aggettivi utilizzati dalla gente per descrivere Giuditta iniziavano con la "i”. Inetta. Indolente. Introversa. Insipida. Imbarazzante. (p. 54) 
Giuditta non pratica nessuna delle attività predilette dalla madre, ma una cosa sa fare: sparare. L'ultima frase del racconto, «E aveva un'ottima mira " lascia intendere che la madre di Giuditta ha poche probabilità di morire serenamente di vecchiaia.

Per arrivare ad Alice nell'ombra, si deve passare attraverso Nemiche, del 1998 : ancora una raccolta di racconti, meno noir ma più cupi, dove le protagoniste sono quindici donne. Figlie, madri, mogli, amanti. Tutte hanno o avrebbero unsistenza normale, se non fosse che la loro vita subisce, come già in $O$ ridere o morire, una sorta di interruzione, una rottura improvvisa che le spinge ad agire e a trasformarsi, ad essere diverse da quello che erano prima, quando erano donne normali, almeno in apparenza. Non sembra che ci sia predestinazione di alcuna sorta, se non nella predisposizione che alcune di loro hanno ad essere nemiche di qualcosa o di qualcuno, o addirittura nemiche fra di loro: qualcuno ha parlato a tale proposito di misoginia, forse per via del testo di Patricia Highsmith; credo tuttavia che la parola misoginia sia poco corretta, giacché mi pare che la misoginia implichi un'avversione anche ideologica nei confronti della donna. Ora, in questo caso, l'odio delle protagoniste non si scatena contro le donne perché donne, è un odio che non si sofferma sulle differenze tra i sessi. Forse è sproporzionato il riferimento alla differenza ontologica così come la sviluppa Vattimo ${ }^{24}$, per esempio, ma la differenza che pure caratterizza tali donne dall'apparenza normale significa che l'essere non è, ma accade. E per riprendere seppur superficialmente il contorto pensiero di Derrida ${ }^{25}$ a tale proposito, la decostruzione temporale che caratterizza la maggior parte dei testi della Garlaschelli non conduce all'essere, ma alla differenza, che è originaria, che è prima di tutto. La differenza è già in queste donne, vittime della spontaneità del loro pensiero e forse della passività della loro intuizione: la differenza spinge queste donne all'azione, molto spesso efferata.

Angela, protagonista del racconto omonimo, quello che chiude il libro, ha due nemiche e un nemico: la madre, Vera; l'amica della madre, Giovanna; il marito, figlio di Giovanna, Maxi. Il racconto si apre su Angela che si sta aggirando in una villa dalle dimensioni abnormi, con un'accetta nella mano destra, alla ricerca della terza vittima, il marito, appunto, che a sua volta la sta cercando, con l'intenzione di fermarla, forse di ucciderla. Angela esplora la casa, immersa nel buio, sempre con l'accetta in mano, e ricorda gli episodi del passato remoto, alternandoli con quelli del passato prossimo. Racconta di sé bambina e del primo 
incontro con Giovanna e Maxi, dopo la scomparsa improvvisa del padre; racconta di come, vent'anni dopo, ritornò a vivere in quella casa e di come Maxi l'amasse, senza mai essere ricambiato. E racconta anche dell'amicizia amorosa che avvolge le due madri, Vera e Giovanna. La protagonista, dopo aver ucciso le due madri, vuole uccidere anche Maxi per riconquistare la libertà, simbolicamente rappresentata da un cappello a falde larghe da sfoggiare senza timore della riprovazione altrui.

In Alice nell'ombra i fatti si precisano: Angela diventa Alice. Non è qui solo una specie di angelo vendicatore che si aggira con l'accetta in una casa somigliante all'hotel Overlook in Shining: Alice ha profondità, carattere, rispetto ad Angela. Alice ha una storia che qui, appunto, viene dettagliata, nel corso dell'esplorazione della casa, alla ricerca di Maxi; insieme a lei, acquistano profondità anche Maxi, e soprattutto le due madri dai nomi cambiati, Sofia, madre di Maxi, ed Elena, madre di Alice.

Alice non è una donna come le altre, avrebbe voluto o potuto esserlo, così come avrebbe potuto essere una bambina come le altre se non avesse o se non avesse avuto una madre come Elena, così dura e tanto ostile. In seguito alla scomparsa del marito, padre affettuoso di Alice, Elena si avvicina all'amica più cara, Sofia, con la quale vive un rapporto quasi simbiotico di amicizia e di amore non ricambiato. Un rapporto che schiavizza, mi scrive Barbara Garlaschelli,

come molto spesso accade con l'amore (e anche di questo volevo raccontare, dell'amore quando diventa una costrizione, quando ti schiaccia perché non sai viverlo). Elena e Maxi amano senza essere ricambiati e forse per questo amano... Sofia e Alice sono imprendibili ma in modo diverso: Sofia basta a se stessa, Alice $\mathrm{no}^{26}$.

Alice accetta di sposare Maxi, indifferente a quello che le succede, lasciandosi scegliere, anche per rimediare al senso di colpa nei confronti della madre:

Sposandomi mi avrebbe resa felice.

Mi amava e solo questo importava, disse una sera [...]. Mi amava, che altro potevo desiderare di più?

Nulla, naturalmente. Non avevo mai desiderato nulla. Non con sufficiente convinzione, almeno. [...] Nessuno mai mi aveva parlato come lui. Nessuno mai mi aveva giurato che si sarebbe preso cura di me.

$\mathrm{E}$ in quel momento mi resi conto di averne disperatamente bisogno. (p. 92)

Alice concede la sua mano e il suo corpo a Maxi, senza mai provare desiderio, senza uno stato d'animo particolare:

Non ha mai smesso di amarmi perché non gli ho mai negato l'unico rifugio nel quale volesse nascondersi: il mio corpo. E come molti uomini innamorati dell'amore, pensò che la mia mente, la mia anima fossero lì, dove c'era il resto di me. (p. 108) 
Lui è ricco, colto, famoso, innamorato, mentre Alice è sempre più indifferente, sterile nelle emozioni e nella sessualità. Solo una volta sembra reagire alla sua stessa passività, con un movimento impercettibile delle dita sulla schiena di Maxi, dita che avrebbero potuto trasformarsi in artigli e che invece si posano sul corpo di lui assecondandone il ritmo:

S’inginocchiò accanto alla poltrona e posò la testa sul mio grembo. La sua mano mi accarezzava la coscia.

"Tesoro, sei così sola, così distante da tutto..."

Sentii la sua mano che scivolava, lenta. Chiusi gli occhi, mandai un sospiro e aprii un poco le gambe. Qualunque cosa purché la smettesse con quel discorso. Mi sollevò il vestito. Sentivo il suo respiro caldo. Lanciavo occhiate verso la porta, aspettandomi da un momento all'altro l'ingresso di mia madre o di Sofia. In pochi istanti mi aveva spogliata e presa, con la voracità di sempre. Affondai le dita nella sua schiena e seguii il suo muoversi sempre più rapido e violento. (p. 110)

L'evocazione delle due madri nel momento dell'intimità contribuisce ad ispessire il senso di soffocamento di cui Alice è vittima: al pieno di lui non sa e non può opporre niente altro che il vuoto della mente e del corpo. E finché le cose continuano così, Alice è solo questo, un corpo vuoto, un corpo senza volto, indifferenziato. Del resto non ha oggetti che la caratterizzino, non ha abiti - ne ha ma è come se non le appartenessero perché non li ha scelti lei-, non ha mobili, non possiede nulla e non ha bisogno di nulla. Ha dei cappelli che prima sua madre e poi Maxi detestano; legge libri che non hanno titolo e vaga al di fuori della grande casa, senza meta, in uno spazio senza immagini.

Un giorno Davide entra nella sua vita e Alice scopre, così, di essere: di essere un corpo, una mente, di avere delle aspirazioni, di provare desiderio e piacere. Scopre di essere libera, viva, scopre di essere diversa da quello che era o che pensava di essere, scopre di poter scegliere: «Davide faceva vivere il mio corpo in sintonia con la mia mente. Non mi era mai accaduto prima. Non sapevo fosse possibile. » (p. 138).

Proprio quando Alice sceglie di vivere amando, proprio quando decide di lasciar emergere la propria differenza - che è la sua essenza di donna giunge ineluttabile la morte, per tutti: si scopre che la madre di Alice ha ucciso il marito, che Maxi ha ucciso Davide, che Alice ha ucciso Sofia ed Elena e che sta aspettando Maxi per uccidere anche lui.

\section{La differenza originale}

La narrazione, in Alice nell'ombra, ha forma di monologo decostruito in tre spazi che dilazionano così lo scorrere del tempo : il passato (dall'infan- 
zia al presente); il presente (Alice che vaga nella casa); la sospensione del tempo (Alice che pensa). Solo così Alice può prendere consistenza, esistere, sottrarsi, ed è proprio qui, in questa decostruzione che appare la differenza di Alice, la differenza originaria. Tra il passato, il presente e l'immanente, grazie proprio all'affioramento della differenza, Alice agisce, spinta da un pensiero estremo e cogente:

Fermarmi, questo devo fare.

Non io che vado incontro a lui, ma lui che viene verso di me.

Non è molto lontano.

Ripete il mio nome.

Alice.

Alice.

Alice.

Lo sento avvicinarsi.

Ha paura, lo so.

$[\ldots]$

Perché ormai adesso so come andrà a finire.

Lui ha l'amore.

Io la rabbia.

E nessuna fretta, ormai. » (p. 184-185)

Lasciando affiorare la differenza primaria, compare nel finale un'Alice molto diversa anche dal personaggio in-differente che attraversa il romanzo in qualità di protagonista e di narratrice apparentemente normale. Mi scrive ancora Barbara Garlaschelli a proposito delle donne «intrappolate in ruoli che spesso non si sentono addosso ": "Vivono vite che non assomigliano a ciò che sono davvero ma non hanno il coraggio di fare scelte diverse, sino a quando qualcosa irrompe nella loro esistenza, sconvolgendole. "

Alice nel paese degli orrori è oramai irriconoscibile, animata come sembra da una sorta di furia omicida, trasformatasi dopo una notte, proprio come avviene all'omonima Alice, nel paese delle meraviglie:

Alice raccolse il ventaglio e i guanti, e perché la sala sembrava una serra si rinfrescò facendosi vento e parlando fra sè : - Povera me! Come ogni cosa è strana oggi! Pure ieri le cose andavano secondo il loro solito. Non mi meraviglierei se stanotte fossi stata cambiata! Vediamo: non son stata io, io in persona a levarmi questa mattina? Mi pare di ricordarmi che mi son trovata un po'diversa. Ma se non sono la stessa dovrò domandarmi: Chi sono dunque? Questo è il problema. - E ripensò a tutte le bambine che conosceva, della sua stessa età, per veder se non fosse per caso una di loro.

- Certo non sono Ada, - disse, - perché i suoi capelli sono ricci e i miei no. Non sono Isabella, perché io so tante belle cose e quella poverina è tanto ignorante! e poi Isabella è Isabella e io sono io. Povera me! in che imbroglio sono! ${ }^{27}$ 
Le due Alici - quella di Carroll e quella della Garlaschelli - sono speculari in tutto, ma entrambe vivono in una dimensione che appartiene solo a loro, fatta di sogni, immagini e pensieri, di solitudine e anche un po'di paura, di fantasia. Ancora, di spontaneità nel pensiero e passività nell'intuizione. Alice, nel paese delle meraviglie, si costruisce, mentre Alice, nel paese degli orrori, si decostruisce, finalmente capace di oltrepassare la soglia della paura che invece l'altra Alice varca fin dall'inizio un po'per sfida, un po' per curiosità. Per accadere.

\section{La differenza siamo noi}

Nell'ultimo libro di Barbara Garlaschelli, intitolato Sorelle, si racconta la storia drammatica e agghiacciante di due sorelle, appunto, Amelia e Virginia, nemiche consanguinee, diverse tra loro eppure profondamente simili, vittime e carnefici di un uomo, Dario, colpevole di aver cercato di sedurle. Si ravvisano qui alcune caratteristiche che rendono peraltro le due sorelle molto simili ad Alice, non foss'altro che tutte e tre, da brave figlie di Meg, all'interno delle spesse pareti di case "nido e prigione ", si consumano nella tragedia che le trasforma da in-differenti a differenti.

E la polizia che fa? Che accade alle Alici scellerate, alle figlie di Meg con la pistola? Credo che spetti al lettore decidere, dato che la polizia, in questi casi, non interviene mai. Come potrebbe, del resto, dipanare queste tragiche matasse? Chi è differente è fuori norma, e quindi fuorilegge. Ma come si fa a trovare il bene ed il male nelle perfide storie di famigliasecondo la formula di Laura Grimaldi ${ }^{28}$ - di fine millenio? Alla fine degli anni'70, Franca Rame concludeva il monologo Una donna sola sulla protagonista che imbraccia il fucile, mentre la cilena Marcela Serrano trasforma la Violeta di Antigua, vita mia, donna dell'epoca neo-democratica, vittima di stupri consumati tra le pareti domestiche, da assassina a giustiziera $^{29}$ : qui le cose sono più semplici, il confine tra bene e male, buono e cattivo, vittime e aguzzini è chiaro, preciso, ben delineato, anche perché queste donne, potenziali figli di Jo in lotta per la loro emancipazione, sono collocabili in una sfera spazio-temporale chiaramente identificata per cui l'assassinio è un vero atto di giustizia.

Le piccole donne assassine, le figlie di Meg con l'accetta in mano, ci mettono in imbarazzo perché la loro differenza, rispetto alla normalità o alla giustizia, non può essere afferrata, definita, delineata chiaramente. Il confine tra normale e non normale è una linea sottile e, come mi scrive ancora Barbara Garlaschelli, 
la normalità non esiste ma è come se molte persone (donne e uomini) ambissero a questa normalità che diventa, però, una sorta di tagliola, di miraggio e di gabbia.

Non ci resta che aspettare, come Alice, l'arrivo di Maxi, consapevoli della differenza originaria che sta in tutti noi, consci del fatto che il trionfo della verità non sempre coincide con il trionfo della giustizia umana. Duca Lamberti, ne I Milanesi ammazzano al sabato ${ }^{30}$, smascherando il colpevole che ha fatto giustizia da sé, malediceva se stesso e imprecava contro il male assoluto che spinge i buoni a diventare spietati assassini. Ma Alice è poi davvero una giustiziera? Non avrebbe potuto trovare altre soluzioni per sfuggire dall'inferno?

In Alice nell'ombra e poi in Sorelle, la realtà è sotto i nostri occhi: spetta ad ognuno di noi decidere in quale verità credere e quale giustizia dovrà prevalere, ché "trionfare " è un verbo che la giustizia regge con difficoltà. E questo il dramma del postmodernismo e del post-postmodernismo: l'enfer c'est les autres, ma in ognuno di noi sta il germe della differenza, che non può essere né volontà né rigetto. C’è, latente.

\section{Notes}

1. Per evitare di cadere negli eccessi dello studio della letteratura per generi, preferisco non utilizzare l'espressione letteratura femminile o al femminile. Vedi E. Rasy, La letteratura e le donne, Roma, Editori Riuniti, 2002. Che mi sia consentito qui di ringraziare Tiziana Jacoponi per i generosi consigli di lettura; grazie anche al mio fraterno amico Ugo Pozzoli per i preziosi suggerimenti filosofico-letterari e per le riletture pazienti.

2. Milano, Salani Editore, 2004, p. 235.

3. M. Serrano, Arrivederci piccole donne, Milano, Feltrinelli, 2005 [2004 per l'edizione originale].

4. Piccole donne (contenente la seconda parte, intitolata Le piccole donne crescono) è scritto e pubblicato tra il 1868 e il 1869; Piccoli uomini è del 1871 e I ragazzi di Jo è del 1886. La prima traduzione in italiano delle Piccole donne dovrebbe risalire al 1918 : da allora, i romanzi della Alcott sono diventati un punto di riferimento per la letteratura infantile destinata alle bambine italiane, figlie del baby boom e appartenenti alla generazione degli "sfiorati". Vedi anche AA. VV., E l'uomo educò la donna, a cura di C. Covato e M. C. Leuzzi, Roma, Editori Riuniti, 1989, contenente un saggio sulla fortuna della Alcott in Italia.

5. <http://www.gutenberg.org/etext/1342>.

6. <http://www.gutenberg.org/etext/514>.

7. L. Campuzano, Las muchachas de La Habana no tienen temor de Dios, L'Avana, Ediciones Unión, 2004.

8. C. Pavese, Tra donne sole [1949], Torino, Einaudi, 1998, p. 60.

9. L. M. Alcott, Piccole donne uccidono, Roma, Editori Riuniti, 1996. Si tratta di una raccolta di racconti della Alcott dedicati al tema della follia e dell'omicidio.

10. B. Garlaschelli, Alice nell'ombra, Milano, Frassinelli, 2002. Le citazioni dal romanzo saranno d'ora in poi indicate semplicemente tra parentesi.

11. Lucía Etxebarría, Noi che non siamo come le altre, Parma, Guanda, 2003 [1999 per l'edizione originale]. La storia di quattro donne inquiete e particolari, indipendenti e bisognose di amore. 
12. S. Bertola, Ne parliamo a cena, Milano, Tea, 1999, p. 200.

13. S. Bertola, Aspirapolvere di stelle, Milano, Tea, 2002, p. 5.

14. S. Bertola, Ne parliamo a cena, op. cit., p. 11.

15. Ibid., p. 23-24.

16. E non è finita... di prossima uscita presso Baldini Castoldi Dalai editore un testo di Lauren Henderson, specialista di Jane Austen, intitolato: Come trovare l'uomo giusto secondo Jane Austen.

17. A. Busi, Cazzi e canguri (pochissimi i canguri), Milano, Frassinelli, 1994.

18. Z. Z. Gábor, A. Busi, Come accalappiare un nomo, come tenerselo stretto e come scaricarlo [1970], Padova, Alet Edizioni, 2005.

19. Così lo descrive lo slogan pubblicitario apparso sulla prima pagina di "Tuttolibri " della Stampa del 12 novembre 2005

20. Cf. L. Etxebarría, Eva futura, Parma, Guanda, 2005 [2000 per l'edizione originale]. Si tratta di un saggio semi serio e poco scientifico sulla questione femminile. Lo cito qui, tuttavia, perché da poco è stato tradotto in italiano, prova del successo editoriale che sta riscuotendo in questi mesi la letteratura « rosa » in Italia, forse anche sulla scia di alcune serie televisive americane, tipo Sex and the city.

Non intendo soffermarmi sulla storia del femminismo o sulle teorie femministe, riguardino esse la letteratura, la sociologia, la giurisprudenza o altri settori: mi è pertanto sembrato inutile fornire qui una bibliografia degli scritti sul femminismo. Rimando per eventuali approfondimenti sul dibattito femminismo dell'uguaglianza e femminismo della differenza all'indispensabile opera di Luce Irigaray ed in particolare a tre saggi particolarmente noti, Ethique de la différence sexuelle, Paris, Éditions de Minuit, 1984; Le temps de la différence, Paris, Librairie générale française, 1989; Je, tu, nous: pour une culture de la différence, Paris, Librairie générale française, 1992. Le traduzioni in italiano dei principali testi di Irigaray sono state curate da Feltrinelli.

21. B. Garlaschelli, Sirena - Mezzo pesante in movimento, Milano, Salani, 2004.

22. Mi pare che esistano significative similitudini tra certi racconti della Garlaschelli, sorprendenti per incisività, e alcuni scritti di Patricia Highsmith, in particolare in Piccoli racconti di misoginia, Milano, Bompiani, 1984 [1977 per l'edizione originale].

23. B. Garlaschelli, O ridere o morire [1995], Lugano, Todaro editore, 2005, p. 99.

24. G. Vattimo, Le avventure della differenza, Milano, Garzanti, 1980.

25. J. Derrida, L'écriture et la différence, Paris, Seuil, 1967.

26. E-mail di Barbara Garlaschelli a Barbara Meazzi, 22 novembre 2005. Ringrazio di cuore Barbara Garlaschelli per aver risposto con pazienza e generosità alle mie domande.

27. <http://www.liberliber.it/biblioteca/d/dodgson/index.htm>

28. L. Grimaldi, Perfide storie di famiglia, Milano, Tropea, 1996.

29. F. Rame, "Una donna sola " [1977], in Le commedie di Dario Fo - Venticinque monologhi per una donna, Torino, Einaudi, 1989, vol. VIII, p. 11-26; M. Serrano, Antigua, vita mia, Milano, Feltrinelli, 2000 [1995 per l'edizione originale].

30. G. Scerbanenco, I Milanesi ammazzano al sabato [1969], Milano, Garzanti, 2001. 